\title{
Three-body forces at work: Three-body potentials derived from triplet correlations in colloidal suspensions
}

\author{
C. Russ ${ }^{1}$, M. Brunner ${ }^{2}$, C. Bechinger ${ }^{2}$ and H. H. von Grünberg ${ }^{3}$ \\ 1 Universität Konstanz, Fachbereich Physik - D-78457 Konstanz, Germany \\ 2 Universität Stuttgart, 2. Physikalisches Institut - D-70550 Stuttgart, Germany \\ 3 Karl-Franzens Universität Graz, Institut für Chemie - A-8010 Graz, Austria
}

PACS. 82.70.Dd - Colloids.

PACS. 82.70.-y - Disperse systems; complex fluids.

PACS. 05.20.Jj - Statistical mechanics of classical fluids.

\begin{abstract}
Two- and three-particle correlation functions are computed from video-microscopy data of two-dimensional suspensions of charged colloids and inverted to derive the pair and three-body interaction potentials between the colloidal particles. Our method allows to resolve the full spatial dependence of the three-body potentials. Examining colloidal systems at different colloid densities, we find density-independent, attractive three-body potentials, with a minimum of a few $\mathrm{kT}$ that is most pronounced in the equilateral triangle configuration.
\end{abstract}

A simple liquid is a liquid consisting of particles which interact with pair potentials only dependent on the particle separations. A charge-stabilized colloidal suspension, by contrast, is a complex fluid: here the inter-particle interaction is governed by the inhomogeneous distribution of electrolyte ions between the highly charged colloids. Only in certain limiting cases, is it possible to integrate out the ionic degrees of freedom, leading to an effective colloid-colloid potential of the Yukawa form. This pair potential is an essential element of the classic DerjaguinLandau-Verwey-Overbeek (DLVO) theory of interactions in charge-stabilized colloids [1,2]. In general, however, the interaction between colloids in a charge-stabilized suspension may not be expected to be pairwise additive: many-body interactions among the colloids can be important, primarily under low salt conditions and for highly charged colloids. A good example is the three-body interaction potential which has received some attention recently: it has been theoretically predicted $[3-5]$, and also experimentally observed $[6,7]$. In $[6,7]$ a video-microscopy experiment is described, examining in what way the presence of a third charged colloid in the neighborhood of a pair of colloids affects their mutual interaction. By decomposing the measured total interaction potential in an appropriate way, the three-particle interaction potentials could be extracted and successfully compared to theoretical Poisson-Boltzmann calculations.

Examining only three isolated particles remains a somewhat artificial situation, considering that, in reality, colloids live together in a suspension of finite density. Therefore, in order to prove that three-body potentials are present also in concentrated colloidal suspensions, one has to observe three-body forces "at work", that is, one has to infer the shape and magnitude of the three-body potential from analyzing the particle coordinates of a large number of colloids which are together in a concentrated suspension. In this letter, we compute correlation functions and extract from these functions the whole three-body interaction potentials among the colloids. Since from two-particle correlations one obtains microscopic information only on the level of pair interactions, we have to analyze pair and, in addition, triplet correlation 
functions, in order to get access to both two- and three-body interaction potentials. At the same time, the present paper aims at presenting an alternative experimental method to measure three-body forces, a method which may be seen to be complementary to the more direct one realized in $[6,7]$.

Using triplet correlations to experimentally determine interaction potentials is possible only in colloidal systems, but cannot be performed in atomic systems in which triplet correlation functions cannot be measured directly. In colloidal suspensions, however, the videomicroscopy technique provides us with hard positional information for each particle at each time-step, which in turn allows us to compute two-, three- and, in principle, even higher-order correlation functions (this is the reason why it has been in a colloidal system that triplet correlation functions have first been observed [8]). We should also remark that our approach is not entirely new, but has in part already been realized by Linse [9]. He simulated asymmetric two-component electrolytes (charge asymmetry $1: 20$ ) within the primitive model and proved the existence of many-body potentials by comparing pair and triplet correlations among colloidal particles in the original two-component and in a reference one-component system. Quite another way of detecting many-body interactions in colloidal systems is to explore their effect on the melting behavior of colloidal crystals [10] which was done using a Poisson-Boltzmann Molecular-Dynamics simulation technique [11].

The paper starts with a brief description of the experimental system, then the numerical method for the structure inversion is introduced and tested, and finally results are presented from applying this method to the experimental data.

Experimental set-up. - The colloidal system under investigation is an aqueous suspension of highly charged sulfate-terminated PS particles of $\sigma=3 \mu \mathrm{m}$ diameter, confined between two glass plates with a $1 \mathrm{~mm}$ spacing. The particles were exposed to vertical light forces which pushed them toward the charged silica plate at the bottom of the cell. So, gravity, electrostatics and light forces confine the colloids to what is an almost perfect two-dimensional system with vertical out-of-plane fluctuations of less than $250 \mathrm{~nm}$. The particle centre positions were analyzed on-line with an imaging processing software. The colloid density was varied by means of optical line traps realized with a sophisticated system of computer-driven mirrors which control a scanned optical laser tweezer. We have investigated six different colloid densities, collected for each density about 1000 colloidal configurations (with up to 1700 particles), and evaluated these data to extract in $[12,13]$ the colloid pair potentials, to compute in [14] the triplet correlation functions and in [15] the equation of state. In all of these papers, we have found at higher densities marked deviations from the expected behavior of a simple Yukawa liquid, in which particles interact via a screened Coulomb potential $u^{(2)}(r)=A \exp [-\kappa r] / r$. In particular, the pair potentials, as derived from the pair correlations, showed a density dependence which in $[12,13]$ we have interpreted in terms of many-body interactions. The present study now corroborates this conclusion by means of three-body potentials explicitly constructed from the very same set of data. We will focus on the three data sets with the highest densities because only they could provide us with an acceptable number of counts for triplets with small inter-particle separations. More details about the experimental set-up and our data analysis can be found in [12].

Inversion procedure. - The three-body potentials are determined in the following way. In the first step, we have to use the video-microscopy data to compute radial pair correlation functions $g^{(2)}(r)$ and triplet correlation functions $g^{(3)}(r, s, t)$ for a particle triplet with distances $r, s$ and $t$ (for details see [14]). The next step is to simultaneously invert both correlation functions, that is, to come from $\left(g^{(2)}(r), g^{(3)}(r, s, t)\right)$ to $\left(u^{(2)}(r), u^{(3)}(r, s, t)\right)$, where $u^{(3)}(r, s, t)$ is the three-particle potential that we are interested in. Here we apply the inverse Monte 
Carlo technique recently suggested by Almarza and Lomba [16] which we have to adapt to our system consisting of particles interacting via $\left(u^{(2)}(r), u^{(3)}(r, s, t)\right)$. Let us assume $g^{(2)}(r)$ and $g^{(3)}(r, s, t)$ are known with their error bars in a discretized form: $\left(r_{i}, g_{i}^{(2)}, \Delta g_{i}^{(2)}\right)$ and $\left(r_{i}, s_{j}, t_{k}, g_{i, j, k}^{(3)}, \Delta g_{i, j, k}^{(3)}\right)$. Here, $i, j, k$ run from 0 to $m-1\left(r_{m}\right.$, in the following, is referred to as the "cut-off" radius). Starting with the first convergence step $\left(l=1, \lambda_{1}=0.1\right)$ we choose as trial functions the potential of mean force for the pair potential $\beta u^{(2)}(r)=-\ln g^{(2)}(r)$ and zero for the triplet potential $\beta u^{(3)} \equiv 0$. After equilibration we take a snapshot of the system and extract the instantaneous correlation functions $\left(g_{\text {inst }}^{(2)}, g_{\text {inst }}^{(3)}\right)$. With these we calculate corrections to our trial potentials from the following expressions:

$$
\begin{aligned}
\beta u_{i}^{(2), \text { new }} & =\beta u_{i}^{(2), \text { old }}+\lambda l \frac{g_{\text {inst }}^{(2)}\left(r_{i}\right)-g_{i}^{(2)}}{\Delta g_{i}^{(2)}}\left\langle\Delta g_{i}^{(2)}\right\rangle, \\
\beta u_{i, j, k}^{(3), \text { new }} & =\beta u_{i, j, k}^{(3), \text { old }}+\mu \lambda_{l} \frac{g_{\text {inst }}^{(3)}\left(r_{i}, s_{j}, t_{k}\right)-g_{i, j, k}^{(3)}}{\Delta g_{i, j, k}^{(3)}}\left\langle\Delta g_{i, j, k}^{(3)}\right\rangle,
\end{aligned}
$$

where $\left\langle\Delta g_{i}\right\rangle$ and $\left\langle\Delta g_{i, j, k}\right\rangle$ are the spatially averaged mean errors. A few cycles of MC steps later, we again extract the instantaneous correlation functions and correct the trial potentials accordingly. (Note that the system does not equilibrate for each new potential.) During each convergence step $l$ the various instantaneous correlation functions are averaged to obtain $\left(g_{l}^{(2)}, g_{l}^{(3)}\right)$. With these averaged correlation functions we check if they resemble sufficiently our reference correlation functions $\left(g_{i}^{(2)}, g_{i, j, k}^{(3)}\right)$ :

$$
\begin{array}{r}
\frac{1}{m} \sum_{i=0}^{m-1}\left(\frac{g_{l}^{(2)}\left(r_{i}\right)-g_{i}^{(2)}}{\Delta g_{i}^{(2)}}\right)^{2} \leq 1, \\
\frac{1}{m^{3}} \sum_{i, j, k=0}^{m-1}\left(\frac{g_{l}^{(3)}\left(r_{i}, s_{j}, t_{k}\right)-g_{i, j, k}^{(3)}}{\Delta g_{i, j, k}^{(3)}}\right)^{2} \leq 1 .
\end{array}
$$

If so, we start convergence step $l+1$. To fulfil both the above criteria, it takes roughly as many trial configurations as there are in the reference data - provided we are using about the same number of particles in both systems (ca 1000). Using less particles (ca 200) sharply increases the number of trial configurations but also significantly reduces computational time without sacrificing accuracy or introducing finite-size effects. In step $l+1$ we decrease $\lambda$ in eq. (1) and eq. (2) according to $\lambda_{l+1}=0.5 \lambda_{l}$ and restart the averaging procedure for the $\left(g_{l}^{(2)}, g_{l}^{(3)}\right)$. While the potentials change dramatically during the first few iteration steps because the system is certainly not equilibrated, we reach equilibration at a later stage $(l=10, \ldots, 15)$. The additional parameter $\mu \approx 0.1, \ldots, 0.01$ in eq. (2) is necessary because $g_{\text {inst }}^{(3)}$ - evaluated from a single frame with a few hundred particles - is very noisy. Therefore, corrections to the triplet trial potential are often stochastic in nature. Introducing $\mu$ we have a parameter with which we can regulate the relative convergence speed of $\left(g_{l}^{(2)}, g_{l}^{(3)}\right)$, respectively $\left(u^{(2)}(r), u^{(3)}(r, s, t)\right)$. Choosing $\mu \approx 0.1$ we allow greater changes for $u^{(3)}$ and therefore relatively faster convergence compared to $u^{(2)}$, by contrast, $\mu \approx 0.01$ means only small changes to $u^{(3)}$ and therefore relatively slow convergence compared to the pair potential.

Inversion accuracy. - To test our method, we performed Monte Carlo simulations with given $u^{(2)}$ and $u^{(3)}$, computed correlation functions and inverted them to see if the resulting potentials reproduced the original input potentials. In the first reference simulation (PairMC) 

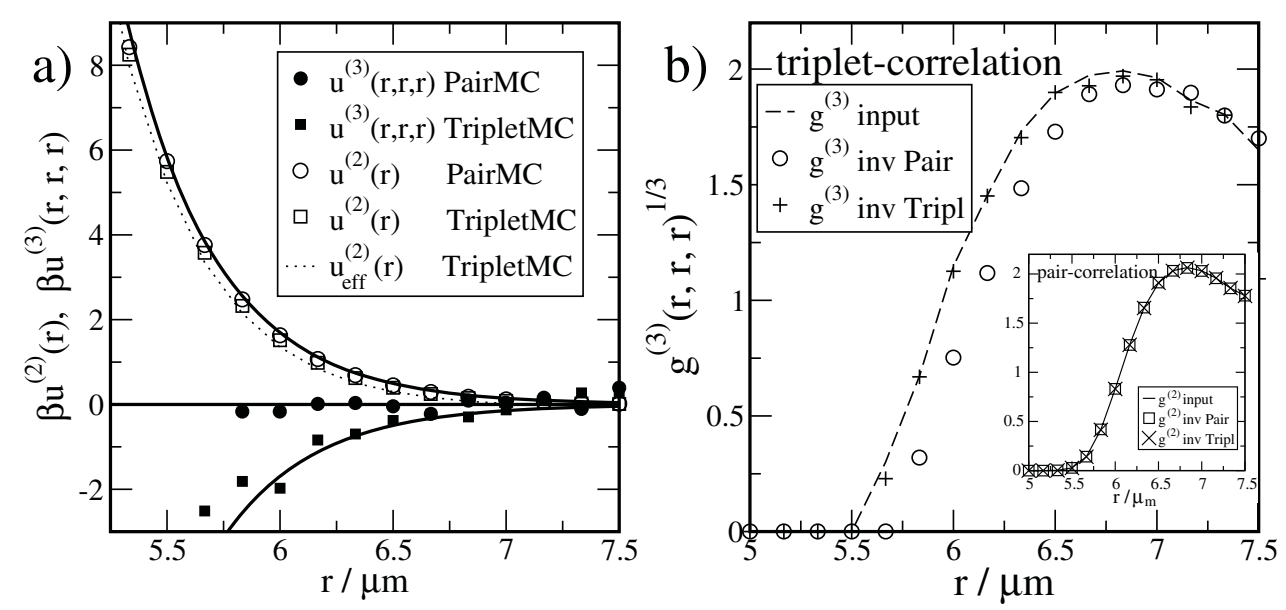

Fig. 1 - Testing the accuracy of our method: a) Reproducing pair and triplet potentials of reference MC simulations with pure pairwise interactions (PairMC) and with pair and triplet interactions (TripletMC). Solid lines are the input potentials of the reference MCs, symbols for the potentials returned by the inversion procedure. b) Pair and triplet correlation function (solid and broken line) from the reference TripletMC simulation, used as input functions in the inversion step. Inverting these correlation functions with only pairwise interactions (inv Pair) leads to an effective potential $u_{\text {eff }}^{(2)}$ (shown in a)) that reproduces $g^{(2)}$ (squares) but fails to recover the correct $g^{(3)}$ (circles). A full triplet inversion (inv Tripl) achieves this (crosses and plus signs).

$u^{(3)}$ was set to zero; so, particles interact in a pairwise fashion with $u^{(2)}(r)=A \exp \left[-\kappa^{(2)} r\right] / r$. In the other simulation (TripletMC), the same repulsive Yukawa pair potentials were combined with an attractive triplet interaction, $u^{(3)}(r, s, t)=-B \exp \left[-\kappa^{(3)}(r+s+t)\right] /(r+s+t)$, where the functional form is inspired from the findings in [5]. Values for $\kappa^{(2)}$ and $A$ are typical experimental values for our systems (for a particle diameter $\sigma=3 \mu \mathrm{m}$ we have $\kappa^{-1}=430 \mathrm{~nm}$ and $Z_{\text {eff }} \approx 16000$ ). To produce strong 3-body effects, $B$ was chosen to be $3 A$ while $\kappa^{(3)}$ was taken to be one third of $\kappa^{(2)}$, so $u^{(2)}$ and $u^{(3)}$ are roughly of the same magnitude and decay on the same length scale. Figure 1a) shows both input functions as solid lines, plotted vs. $r$ and $r=s=t$ (side length of an equilateral triangle). The symbols give the potentials we obtain after the inversion, demonstrating that, for both simulations, the input potentials are indeed successfully reproduced.

It is interesting to invert the TripletMC simulation (that is, the simulation including threebody potentials!) also on a pairwise level, i.e., such that the inversion determines just $u^{(2)}(r)$. Then the effect of the three-body interaction is absorbed entirely into what is known as an effective pair potential $u_{\text {eff }}^{(2)}$ (dotted line in fig. 1a)). Pair potentials such as these are then density dependent; for the data herein, they were analyzed in $[12,13]$. However, one should keep in mind that using these effective potentials one can reproduce the micro-structure of the fluid only on the level of pair correlations. This is evident from fig. 1b) showing $g^{(3)}(r, r, r)$ as obtained from the TripletMC simulation, and in the inset $g^{(2)}(r)$. Both functions are inverted in two different ways: i) by allowing for a full determination of $\left(u^{(2)}(r), u^{(3)}(r, s, t)\right)$ (labelled "inv Tripl" in the figure) and ii) by allowing only for an effective pair potential $u_{\text {eff }}^{(2)}$ ("inv Pair" in fig. 1b)). While in both cases $g^{(2)}$ at the end of the inversion agrees perfectly with the $g^{(2)}$ put into the inversion routine (see the inset of fig. 1b)), $g^{(3)}$ is properly reproduced only if the inversion allows for three-body potentials. This important observation justifies our whole approach, that is, $\left(g^{(2)}(r), g^{(3)}(r, s, t)\right) \rightarrow\left(u^{(2)}(r), u^{(3)}(r, s, t)\right)$. 


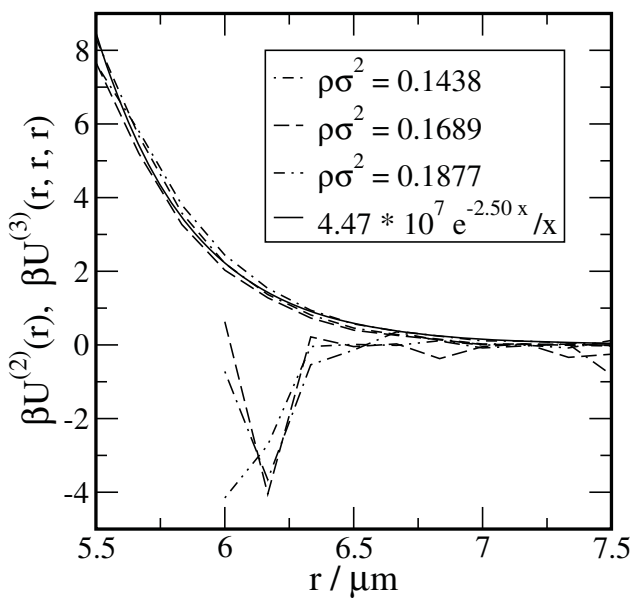

Fig. 2 - Pair potentials (upper curves) and three-body potentials (lower curves) for three different colloid densities, as a function of the radius $r$ and the side length of an equilateral triangle $r=s=t$, respectively, obtained from the inversion of pair and triplet correlation functions in $2 \mathrm{D}$ colloidal suspensions. The solid curve is a potential fitted to the experimental pair potentials.

Experimental results. - We now apply our method to the experimental data of $[12,13]$. Typical triplet correlation functions as produced from these data are plotted in [14]. In fig. 2 we show the pair and triplet potentials obtained from inverting the correlation functions for the three highest densities investigated in $[12,13]$. One observes density-independent pair potentials with a slight shift due to a small drift in the salt concentration over time. The triplet potentials are also (roughly) density independent and show an attractive well of about $4 \mathrm{kT}$ for very short distances. Having tested our method thoroughly against various MC simulations with purely pairwise interactions we have made sure that this minimum is no artifact of our inversion procedure. We now take a closer look at $u^{(3)}$ choosing the density $\rho \sigma^{2}=0.1438$ that provides us with the best statistical accuracy. Figure 3 shows the full $s$ and $t$-dependence of the three-body potential for three different values of $r$. The potentials are cut below $s_{\min }=t_{\min }=6 \mu \mathrm{m}$, where an inversion becomes impossible due to the limited statistics in $g^{(3)}$. For the smallest value of $r$ in fig. 3a) an extended minimum of up to $4 \mathrm{kT}$ depth is observed which seems to be centred at $r=s=t=6 \mu \mathrm{m}$. For a slightly larger

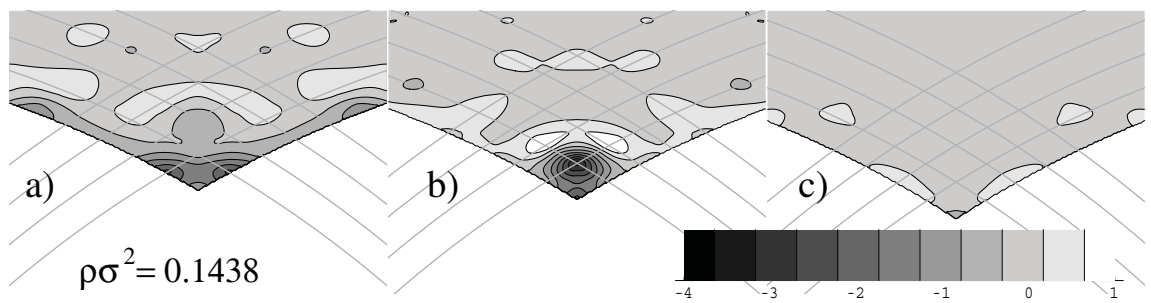

Fig. 3 - Potential landscape of three-body potential of the $\rho \sigma^{2}=0.1438$ measurement, for a) $r=$ $6.0 \mu \mathrm{m}, \mathrm{b}) r=6.167 \mu \mathrm{m}$ and c) $r=6.5 \mu \mathrm{m}$. Two colloids (relative distance $r$ ) are held fixed at the centres of the concentric white circles which define lines of constant $s$ and $t$, where the third colloid can be located. The grey-level scheme is in steps of $0.5 \mathrm{kT}$, the mesh size is $\Delta s=\Delta t=0.167 \mu \mathrm{m}$, used also in the inversion procedure. The potentials are cut below $s_{\min }=t_{\min }=6 \mu \mathrm{m}$. 
value of $r(r=6.167 \mu \mathrm{m})$ in $\mathrm{b})$, one clearly recognizes that the three-body attraction is most pronounced in the region near the vertex where $r=s=t$ (cf. fig. 2). Increasing $r$ further any triplet contribution quickly vanishes as seen in fig. 3c).

The three-body potential has a minimum of $-4 \mathrm{kT}$ at a distance where the pair energy is around $1 \mathrm{kT}$, but nevertheless the effect of this $u^{(3)}$ on $g^{(3)}$ is rather weak. Producing a plot such as fig. 1b), we see an almost negligible difference between the triplet correlation functions resulting from the pairwise and the full triplet inversion. This is due to the fact that the mean distance between neighboring particles is around 6.6 to $7 \mu \mathrm{m}$. Triplets with particle separations of $6.167 \mu \mathrm{m}$ and less are therefore rather rarely sampled and (looking at fig. 3c)) already at $r=6.5 \mu \mathrm{m}$ - less than the typical pair distance - no triplet contribution can be seen no matter how small $s$ and $t$ are. Because of the undersampling of $g^{(3)}$ at $r=s=t=6.0 \mu \mathrm{m}$, the potentials at these distances in figs. 2 and 3 should be interpreted with some caution; so, the upswing of two curves at $r=s=t=6.0 \mu \mathrm{m}$ in fig. 2 is probably unphysical [17].

Evidently, three-body potentials are attractive and most pronounced in the equilateral triangle configuration, which is both in line with theoretical [5] and experimental findings [6]. They are observed only when all three colloids are very close to each other, at distances much smaller than the mean particle distance. For geometrical reasons, we can rule out that the interaction of a colloidal triplet in a $2 \mathrm{D}$ system at such a small value of $r=s=t$ is influenced by a fourth or fifth colloidal particle in the neighborhood. While in general our method produces three-body interaction potentials which are effective in the sense that they include also higher-order interactions, we here, for the particular case at hand, can conclude from the special distance dependence that just three-body interactions, but no higher-order interactions, are present.

Two further points should be noted. Firstly, our method of deriving three-body potentials from correlation functions provides us with the full spatial dependence of the potentials, while in $[6,7]$ we could measure three-body potentials only in one fixed geometry. Secondly, fig. 2 confirms that we now obtain density-independent potentials, as opposed to the results of the pure pair correlation analysis presented in [12,13], where we have found density-dependent, but effective, pair potentials. However, we hasten to add that the density dependence of the effective pair potentials discussed in $[12,13]$ is only partly, but not entirely, explained by the three-body potentials from fig. 2. A rather artificial, additional contribution results from the inversion procedure which depends sensitively on the cut-off as pointed out in [18]. Here we have chosen the cut-off after performing test calculations such as in fig. 1; the best choice turned out to be a cut-off directly behind the first maximum of $g^{(2)}$ and $g^{(3)}$, just where $u^{(2)}$ becomes zero (choosing a significantly larger cut-off, the inversion procedure produced densitydependent minima even in cases where the PairMC simulation data were used!). Despite all this, the main conclusion of $[12,13]$, the existence of many-body interactions in chargestabilized colloidal suspensions, is clearly confirmed by the present study.

Conclusion. - In order to explicitly prove that three-body forces are present in chargestabilized colloidal suspensions, we have analyzed the micro-structure of a colloidal liquid on the level of triplet correlations to get access to the three-body interaction potentials among the colloidal particles. We have demonstrated with fig. 1 that effective pair potentials $u_{\text {eff }}^{(2)}$, if appropriately chosen, can produce the correct $g^{(2)}$, but not the correct $g^{(3)}$. Triplet correlations are thus clearly seen to be needed to determine unambiguously three-body interactions from the colloidal micro-structure. We have applied our inversion procedure to the experimental data from $[12,13]$, and found attractive three-body potentials of about $-4 \mathrm{kT}$ in depth, most pronounced in the equilateral triangle configuration, but at a distance which is too short (and thus too rarely realized) to strongly influence the micro-structure. 
Ideally, in order to produce comparable results, one should apply our method to suspensions made of the same particles as used in $[6,7]$. But these particles turned out to be unsuitable to form stable $2 \mathrm{D}$ suspensions. There is work in progress using smaller particles since they would bring us closer to the parameters studied in $[5,19]$.

We gratefully acknowledge stimulating suggestions and a careful reading of the manuscript by Dr. J. Dobnikar, Karl-Franzens Universität Graz, and Dr. P. Royall, Utrecht University. This work has been supported by the Deutsche Forschungsgemeinschaft, SFB 513 Konstanz.

\section{REFERENCES}

[1] Verwey E. J. W. and Overbeek J. T. G., Theory of the Stability of Lyophobic Colloids (Elsevier, Amsterdam) 1948.

[2] Löwen H. and Hansen J. P., Annu. Rev. Phys. Chem., 51 (2000) 209.

[3] Löwen H. and Allahyarov E., J. Phys. Condens. Matter, 10 (1998) 4147.

[4] Wu J. Z., Bratko D., Blanch H. W. and Prausnitz J. M., J. Chem. Phys., 113 (2000) 3360.

[5] Russ C., van Roij R., Dijkstra M. and von Grünberg H. H., Phys. Rev. E, 66 (2002) 011402 .

[6] Brunner M., Dobnikar J., von Grünberg H. H. and Bechinger C., Phys. Rev. Lett., 92 (2004) 078301.

[7] Dobnikar J., Brunner M., von Grünberg H. H. and Bechinger C., Phys. Rev. E, 69 (2004) 031402.

[8] Zahn K., Maret G., Rub C. and von Grünberg H. H., Phys. Rev. Lett., 91 (2003) 115502.

[9] Linse P., J. Chem. Phys., 94 (1991) 8227.

[10] Dobnikar J., Rzehak R. and von Grünberg H. H., Europhys. Lett., 61 (2003) 695; Dobnikar J., Chen Y., Rzehak R. and von Grünberg H. H., J. Phys. Condens. Matter, 15 (2002) S263; J. Chem. Phys., 119 (2003) 4971.

[11] Fushiki M., J. Chem. Phys., 97 (1992) 6700; Löwen H., Hansen J. P. and Madden P. A., J. Chem. Phys., 98 (1993) 3275; Dobnikar J., Haložan D., Brumen M., von GrünberG H. H. and Rzehak R., Comput. Phys. Commun., 159 (2004) 73.

[12] Klein R., von Grünberg H. H., Bechinger C., Brunner M. and Lobaskin V., J. Phys. Condens. Matter, 14 (2002) 7631.

[13] Brunner M., Bechinger C., Strepp W., Lobaskin V. and von Grünberg H. H., Europhys. Lett., 58 (2002) 926.

[14] Rub C., Zahn K. and von Grünberg H. H. J. Phys. Condens. Matter, 15 (2003) S3509.

[15] Brunner M., Bechinger C., Herz U. and von Grünberg H. H., Europhys. Lett., 63 (2003) 791.

[16] Almarza N. G. and Lomba E., Phys. Rev. E, 68 (2003) 011202.

[17] In $[6,7]$ the undersampling problem could be circumvented by strongly pushing together the colloidal particles by means of external light forces.

[18] Lobaskin V., Brunner M., Bechinger C. and von Grünberg H. H., J. Phys. Condens. Matter, 15 (2003) 6693.

[19] In [5] we used scaled units and studied the regime $\bar{Z}=0.34, \ldots, 9.0$ for the scaled colloidal charged, and $\bar{\kappa}^{-1}=0.39, \ldots, 6.25$ for the scaled screening length. In the present paper we have $\bar{Z}=6.7$ and $\bar{\kappa}^{-1}=0.13$, which is outside the range studied in [5]. For these parameters, our Poisson-Boltzmann solver fails to produce results accurate enough to compute three-body potentials. 